\title{
PRESENCIA DE ANTICUERPOS CONTRA Borrelia burgdorferi Y Anaplasma sp EN CANES DE LA CIUDAD DE LIMA
}

\author{
Presence of Antibodies against Borrelia burgdorferi And Anaplasma SP IN \\ dogs in Lima, Peru
}

Alicia M. Rubio ${ }^{1,2}$, Elia A. Salas ${ }^{1,3}$, Gisella Gómez ${ }^{4}$

\section{Resumen}

Dos perros fueron diagnosticados con borreliosis y uno con anaplasmosis, ambos casos hallados por primera vez en la ciudad de Lima, Perú. Los animales presentaban signos inespecíficos como decaimiento y palidez de mucosas, y uno de ellos con petequias y equimosis en piel. El diagnóstico de laboratorio se hizo mediante una prueba de ELISA comercial.

Palabras clave: borreliosis, anaplasmosis, Snap 4Dx

\section{Abstract}

Two dogs were diagnosed with Lyme borreliosis and one dog with anaplasmosis. The animals showed unspecific clinical signs like depression, pale mucose membranes, and one of them had petechiae and ecchymotic skin lesions. The laboratory diagnose was done by an ELISA commercial test. This is the first report of these diseases in Lima, Peru.

Key words: Lyme borreliosis, anaplasmosis, Snap 4Dx

\section{INTRODUCCIÓN}

La borreliosis es una infección producida por espiroquetas del género Borrelia que afecta al hombre y a los animales (Glenny et al., 2004). Es transmitida a los perros a través de garrapatas pertenecientes al complejo
Ixodes; siendo el $I$. ricinus el vector más común en la transmisión de la infección en Europa y el I. scapularis e I. pacificus en América (Dickinson y Batlle, 1997). El signo clínico más frecuente de la borreliosis o enfermedad de Lyme es la artritis, signo muy similar al producido por A. phagocytophilum; sin embargo, también se puede pre-

\footnotetext{
${ }^{1}$ Consultorio Veterinario Alicia Rubio, Lima, Perú

${ }^{2}$ E-mail: alicia@veterinariarubio.com.pe

${ }^{3}$ E-mail: eliasalasc@yahoo.com

${ }^{4}$ Representante de IDEXX Perú
} 
sentar anorexia, fiebre, letargo, dolor inespecífico, o signos cutáneos, oculares, renales, cardiacos, y desórdenes neurológicos debido a la localización tisular de la bacteria (Wiebe, 1995; Alleman y Sayler, 2010).

La anaplasmosis canina es causada por bacterias gram negativas, de crecimiento intracelular obligado. Estas son Anaplasma phagocytophilum y A. platys (Alleman y Wamsley, 2008). La infección por la primera es la más comúnmente asociada con anaplasmosis, produciendo cojera y siendo confundida usualmente con la Enfermedad de Lyme o borreliosis; mientras que la segunda ocasiona una trombocitopenia cíclica infecciosa en el perro, debido a la infección intracelular en las plaquetas (Alleman y Couto, 2006). Anaplasma platys fue anteriormente conocida como Ehrlichia platys, pero en el 2001 fue reclasificada dentro de la familia Anaplasmataceae, género Anaplasma (Dumler et al., 2001).

Varias garrapatas del genero Ixodes pueden servir como vectores de $A$. phagocytophilum e infectar a una amplia gama de mamíferos, incluyendo perros, gatos, humanos y especies silvestres (Breitschwerdt, 2006). El agente vector de A. platys no ha sido claramente determinado, pero se ha identificado su ADN en garrapatas de los géneros Dermacentor y Riphicephalus (Alleman y Wamsley, 2008). Rhipicephalus sanguineus es la garrapata transmisora de Erhlichia canis y se sospecha que también actúa como vector de $A$. platys (Alleman y Sayler, 2010).

Los signos clínicos de la anaplasmosis y erhichiosis en el perro son inespecíficos, pudiéndose encontrarse individuos asintomáticos. A. phagocitophylum puede causar fiebre, linfoadenomegalia, letargia, hinchazón y dolor articular, signos neurológicos y hemorragias, pudiendo llegar a ocurrir la muerte (Alleman y Sayler, 2010). Asimismo, ocurre trombocitopenia, linfopenia y elevación de las transaminasas (Correia Marques da Silva, 2010). En el caso de A. platys, la trombocitopenia se deriva en un bacteremia y trombocitopenia cíclica de 10 a 14 días de intervalo, anemia no regenerativa, leucopenia e hipoalbuminemia (Alleman y Wamsley, 2008).

El diagnóstico de estas enfermedades se determina a través los signos clínicos presentados en el paciente, conjuntamente con las alteraciones hematológicas y bioquímicas encontradas. Además, se utilizan pruebas serológicas rápidas en la práctica clínica diaria debido a su fácil manejo y rapidez en el diagnóstico, como la prueba de ELISA SNAP ${ }^{\circledR} 4 \mathrm{Dx} \circledast$ (Idexx Laboratories), y a través de pruebas moleculares capaces para identificar secuencias específicas de ADN, como la prueba de PCR (Hoyos et al., 2007).

\section{Materiales y Métodos}

Se describe el caso de tres caninos que llegaron a consulta por diferentes motivos y en fechas distintas. Los tres animales fueron sometidos a un examen clínico completo, además de análisis de laboratorio. Los datos históricos de mayor relevancia se encuentran en el Cuadro 1.

A los pacientes 1 y 3 se les realizó hemograma completo, bioquímica sanguínea básica (ALT, fosfatasa alcalina, BUN, creatinina, glucosa, proteínas totales, albúmina y globulina), urianálisis y ecografía abdominal. Al paciente 2 solo se le practicó un hemograma completo. Los valores de los análisis clínicos fuera del rango referencial se muestran en el Cuadro 2.

Se colectó muestras de sangre a los tres pacientes y se realizó la prueba ELISA SNAP ${ }^{\circledR} 4 \mathrm{Dx}{ }^{\circledR}$, prueba de diagnóstico in vitro para la detección del antígeno de Dirofilaria immitis y anticuerpos contra $A$. phagocytophilum, Borrelia burgdorferi y Ehrlichia canis en suero, plasma o sangre entera de caninos (IDEXX Laboratories Inc). 
Cuadro 1. Datos clínicos de tres canes con sospecha de patógenos hemáticos

\begin{tabular}{|c|c|c|c|}
\hline & Paciente 1 & Paciente 2 & Paciente 3 \\
\hline Raza & Shih tzu & Jack Russel Terrier & Cocker Inglés \\
\hline Sexo y edad & Hembra, 5 años & Macho, 2 años & Macho, 11 años \\
\hline Procedencia & $\begin{array}{l}\text { Recogida, vive en } \\
\text { Santa Anita }\end{array}$ & San Martín de Porres & $\begin{array}{l}\text { Llegó de EEUU un año } \\
\text { atrás, vive en S. Isidro }\end{array}$ \\
\hline $\begin{array}{l}\text { Historia de } \\
\text { garrapatas }\end{array}$ & Nunca vistas & $\begin{array}{l}15 \text { días antes de la } \\
\text { consulta }\end{array}$ & $\begin{array}{l}\text { Nunca vistas en EEUU } \\
\text { ni en Perú }\end{array}$ \\
\hline $\begin{array}{l}\text { Motivo de } \\
\text { consulta }\end{array}$ & $\begin{array}{l}\text { Decaimiento } \\
\text { generalizado }\end{array}$ & Laceración en piel & $\begin{array}{l}\text { Decaimiento, anorexia } \\
\text { y heces pastosas }\end{array}$ \\
\hline $\begin{array}{l}\text { Hallazgos } \\
\text { clínicos }\end{array}$ & $\begin{array}{l}\text { Inespecíficos, } \\
\text { decaimiento general }\end{array}$ & $\begin{array}{l}\text { Petequias y equimosis } \\
\text { en piel }\end{array}$ & $\begin{array}{l}\text { Palidez de } \mathrm{MM}^{1} \text {, } \\
\text { decaimiento general }\end{array}$ \\
\hline $\begin{array}{l}\text { Fecha del } \\
\text { análisis }\end{array}$ & Enero 2010 & Febrero 2010 & Mayo 2010 \\
\hline
\end{tabular}

${ }^{1} \mathrm{MM}=$ Membranas mucosas

Cuadro 2. Valores de análisis clínicos fuera del rango referencial en tres canes con sospecha de patógenos hemáticos

\begin{tabular}{|c|c|c|c|c|}
\hline Análisis realizado & Paciente 1 & Paciente 2 & Paciente 3 & Valor de referencia \\
\hline \multicolumn{5}{|l|}{ Hemograma } \\
\hline - Hematocrito, $\%$ & 48.7 & 41.7 & 29.50 & $37.0-55.0$ \\
\hline - Hemoglobina, g/dl & 17.5 & 13.7 & 10.0 & $12.0-18.0$ \\
\hline - Plaquetas, /ml & 223,000 & 29,000 & 103,000 & $175,000-500,000$ \\
\hline - Leucocitos, /ml & 10,800 & 24,500 & 15,800 & $6,000-16,900$ \\
\hline - Neutrófilos, ml & 8,800 & 20,200 & 12,700 & $3,300-12,000$ \\
\hline \multicolumn{5}{|l|}{ Bioquímica } \\
\hline - Albúmina, g/dl & 3.5 & & 2.2 & $2.3-4.0$ \\
\hline - Globulina, g/dl & 6.2 & & 4.8 & $2.5-4.5$ \\
\hline Ecografía & Esplenomegalia & Normal & $\begin{array}{l}\text { Esplenomegalia, } \\
\text { masa intestinal }\end{array}$ & \\
\hline \multicolumn{5}{|l|}{ Urianálisis } \\
\hline - Proteinuria & Trazas & & $1+$ & Negativo \\
\hline
\end{tabular}


Cuadro 3. Resultados a la prueba ELISA SNAP® ${ }^{\circledR}$ Dx ${ }^{\circledR}$ (IDEXX Laboratories Inc)

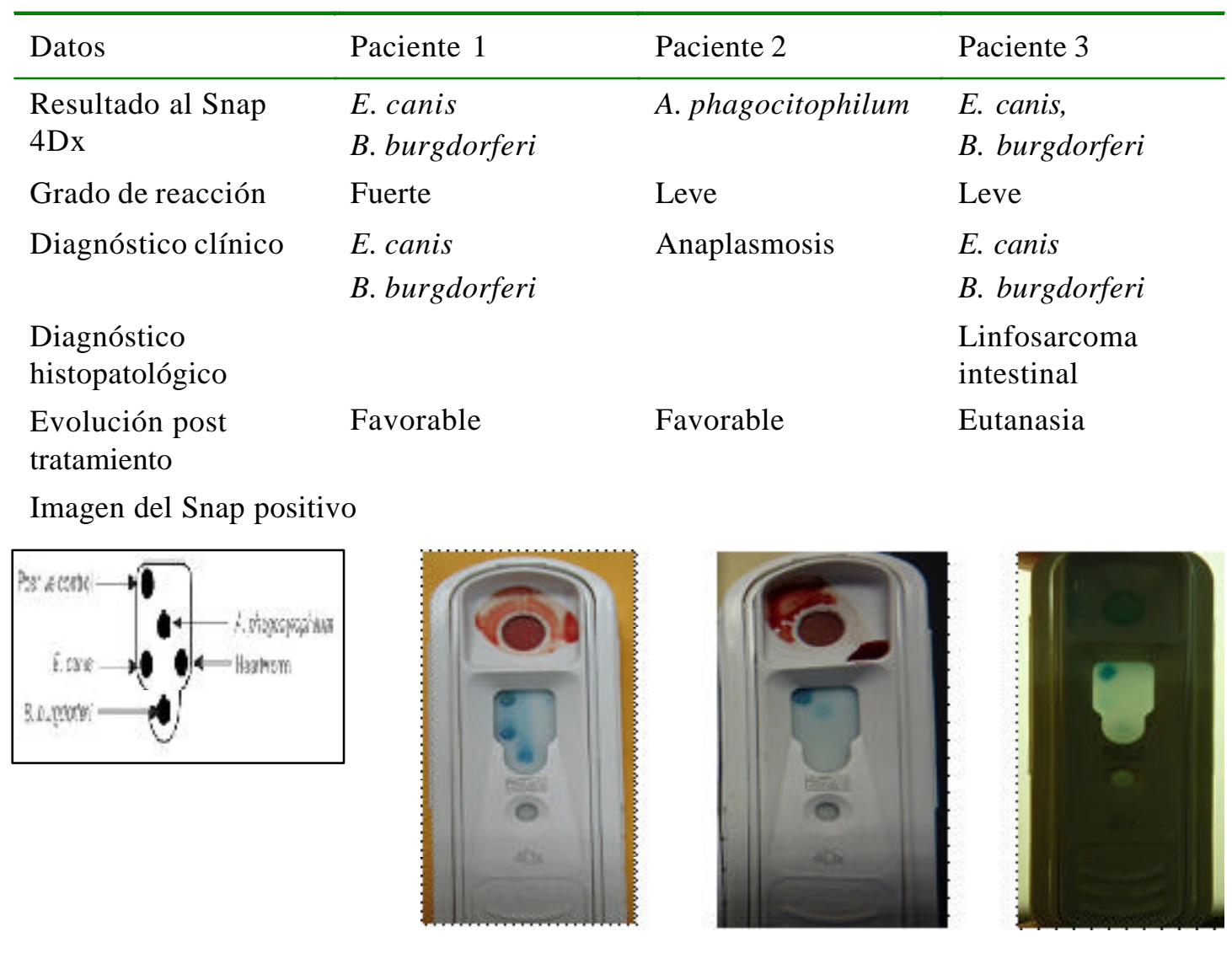

\section{Resultados y Discusión}

Los resultados de la prueba ELISA se muestran en el Cuadro 3. Los pacientes 1 y 3 resultaron positivos a $B$. burgdorferi. En el Perú, la borreliosis de Lyme ha sido diagnosticada en humanos en los departamentos de Piura y Amazonas, donde ha sido reportada la presencia del vector (Macedo et al., 1994; Glenny et al., 2004); sin embargo, anticuerpos contra esta bacteria no han sido reportados en perros en el país. Se desconoce dónde pudieron haber entrado en contacto con el vector, ya que el paciente 1 fue recogido de la calle en el distrito de Santa Anita, Lima, y el paciente 3 nació y vivió en EEUU por varios años, teniendo sólo un año de residencia en Lima.
Las garrapatas del género Ixodes son prevalentes en varias zonas de EEUU y Europa, encontrándose en áreas boscosas (Ostfeld, 1997). En el Perú, se les encuentra parasitando perros, gatos y cerdos y aunque aún no se han reportado la presencia de este género en la ciudad de Lima, se le ha encontrado en varias localidades de Piura (Glenny et al., 2004) y, también, en vizcachas en Loreto y San Martín (Macedo et al., 1994); zonas que cumplen con las condiciones medioambientales adecuadas para el cumplimiento del ciclo biológico del parásito.

Los pacientes 1 y 3 dieron, además, reacción positiva a anticuerpos contra Ehrlichia canis. Esta coinfección de dos agentes en un mismo individuo tiene particular interés, toda vez que $B$. burdogferi y $E$. 
canis son transmitidas por garrapatas del género Ixodes y Rhipicephalus, respectivamente, lo que indica que ambos individuos estuvieron expuestos a los dos vectores en algún momento de su vida.

El paciente 2 fue positivo a $A$. phagocytophilum y el propietario reportó la presencia de garrapatas dos semanas previas al examen (Cuadro 1); sin embargo, no se encontró este vector durante la revisión clínica. Se conoce que el kit diagnóstico empleado tiene reacción cruzada con $A$. platys (Alleman y Sayler, 2010), y que este patógeno ha sido reportado en diversas regiones del continente (Breitschwerdt, 2006). Por otro lado, $R$. sanguineus, posible vector de $A$. platys se encuentra comúnmente en varios distritos de Lima (Bustamante, 1998), motivo por el cual esta garrapata podría haber sido el vector transmisor en este caso. No obstante, anticuerpos contra Anaplasma no han sido reportados en el Perú.

Estas enfermedades pueden causar la aparición de signos clínicos o los animales pueden permanecer como portadores con una infección subclínica durante un periodo de tiempo prolongado (Alleman y Sayler, 2010). Los pacientes 1 y 3 fueron portadores de $B$. burdogferi y E. canis, pero no se reporta historia de garrapatosis en ninguno de los dos casos, por lo que no se conoce el posible momento de la infección. Los portadores subclínicos de estos agentes pueden llegar a presentar signos de la enfermedad cuando hay situaciones de estrés o inmunodepresión (Alleman y Couto, 2006). El cuadro neoplásico encontrado en el paciente 3 pudo haber contribuido en la manifestación de los signos clínicos de estos agentes. El animal fue eutanasiado días posteriores de haberlo sometido a una laparotomía exploratoria con una parcial enterectomia, teniéndose el diagnóstico histológico de la masa localizada en el intestino como linfoma intestinal.
Los pacientes 1 y 2 fueron tratados con doxiciclina a razón de $10 \mathrm{mg} / \mathrm{kg} /$ día, y tuvieron una evolución clínica favorable.

La ehrlichiosis canina es prevalente en la ciudad de Lima (Adrianzen et al., 2003; Hoyos et al., 2007), de allí que es la primera patología que se considera en pacientes con signos clínicos y hallazgos de laboratorio que sugieran la presencia de este agente; sin embargo, los resultados presentados en este reporte demuestran la existencia de anticuerpos contra Anaplasma y Borrelia en esta cuidad y, por lo tanto, habría que considerarlas en el diagnóstico diferencial; especialmente en áreas donde la exposición a las garrapatas es alta, y cuando se presenta un malestar inespecífico de fiebre y letargia, sin bioquímicas importantes y solo anormalidades hematológicas, como en los tres casos presentados. Por otro lado, se deben realizar estudios adicionales con técnicas como inmunofluorescencia indirecta (IFI) y la reacción en cadena de la polimerasa (PCR) para dilucidar la presencia de estos patógenos en la ciudad de Lima.

\section{Literatura Citada}

1. Adrianzen J, Chávez A, Casas E, Li O. 2003. Seroprevalencia de la dirofilariosis y ehrlichiosis canina en tres distritos de Lima. Rev Inv Vet, Perú 14: 43-48.

2. Alleman R, Couto G. 2006. Testing for tick borne diseases: How and when? [Internet]. Disponible en: http:// www.vet.ohio-state.edu/assets/pdf/hospital/bloodBank/wellness/research/ TestingforTickBorneDiseases.pdf

3. Alleman $R$, Sayler $K .2010$. Coinfección en las enfermedades transmitidas por vectores: la perspectiva en los EEUU. Boletín Idexx Laboratorios SL. c/ plom n ${ }^{\circ} 2-8,3^{\circ}$. [Internet]. Dis- 
ponible en: http://www.idexx.es/ saludanimal/education/

4. Alleman R, Wamsley HL. 2008. An update on anaplasmosis in dogs. [Internet]. Disponible en: http:// veterinarymedicine.dvm360.com/ vetmed/Parasitology+Center/Anupdate-on-anaplasmosis-in-dogs/ ArticleStandard/Article/detail/506867

5. Breitschwerdt E. 2006. Question related to interpretation of the IDEXX Snap 4Dx. [Internet]. Disponible en: http://www.cvm.ncsu.edu/vth/.../ interpretation_SNAP_4DX_1_07.pdf

6. Bustamante A. 1998. Prevalencia de ectoparásitos en Canis familiaris en la zona climática litoral de Lima Metropolitana en la estación de invierno. Tesis Médico Veterinario. Lima: Facultad de Medicina Veterinaria, Universidad Nac Mayor de San Marcos. 43 p.

7. Correia Marquez da Silva E. 2010. Ehrilichia canis, Rickettsia conorii e Anaplasma phagocytophilum. Estudo da prevalencia por IFI y PCR em populacao canina da área metropolitana do Porto. Tesis de Maestría. Portugal: Instituto de Ciencias Biomedicas Abel Salazar, Universidad do Porto. 6 p.

8. Dickinson M, Batlle M. 1997. Borreliosis de Lyme: acercamiento a una enfermedad infecciosa emergente. Rev Cubana Hig Epidemiol 35(2): 94-105.

9. Dumler JS, Barbet AF, Bekker CP, Dasch GA, Palmer GH, Ray SC,
Rikihisa Y, Rurangirwa FR. 2001. Reorganization of genera in the families Rickettsiaceae and Anaplasmataceae in the order Rickettsiales: unification of some species of Ehrlichia with Anaplasma, Cowdria with Ehrlichia and Ehrlichia with Neorickettsia, descriptions of six new species combinations and designation of Ehrlichia equi and 'HGE agent' as subjective synonyms of Ehrlichia phagocytophila. Int J Syst Evol Micr 51: 2145-2165.

10. Glenny M, Mendoza L, Falconi E. 2004. Detección de anticuerpos contra Borrelia burgdorferi e identificación de garrapatas ixodidas en Piura y Amazonas, Perú. Rev Per Med Exp Salud Pública 20(1): 23-27.

11. Hoyos L, Li O, Alvarado A, Suarez F, Díaz D. 2007. Evaluación del examen hematológico en el diagnóstico de Ehrlichiosis canina. Rev Inv Vet, Perú 18: 129-135.

12. Macedo AS, Cuadra L, Arevalo J, Jove I. 1994. Borreliosis de Lyme: Probables vectores implicados en la transmisión del agente etiológico. Rev Per Med Trop UNMSM 8(1-2): 27-32.

13. Ostfeld R. 1997. The ecology of Lyme Disease risk. American Scientist. 85: 338-346

14. Wiebe C. 1995. Canine Lyme borreliosis in Ontario - A case report. Can Vet J 36: 513-514. 